\title{
FINITE NUMBER OF DOUBLE COSETS IN A FREE PRODUCT WITH AMALGAMATION ${ }^{1}$
}

\author{
DRAGOMIR Ž. DJOKOVIĆ
}

\begin{abstract}
If $\boldsymbol{H}$ is a finitely generated subgroup of a free group $\boldsymbol{G}$ such that every conjugate of $H$ contains a cyclically reduced word then $(G: H)<\infty$. This generalizes a well-known result of Karrass and Solitar. If $H$ is a finitely generated subgroup of the free product with amalgamation $G=A * U B$ such that every conjugate of $H$ meets $A$ and $B$ trivially and contains a cyclically reduced word then $G$ has only finitely many $(H, U)$-double cosets. Both theorems are proved by defining an action of $G$ on a tree such that $H$ acts freely.
\end{abstract}

1. Statement of results. The object of this note is to prove the following two theorems.

THEOREM 1. Let $H$ be a subgroup of a free group $G$ such that every conjugate of $H$ contains a cyclically reduced word. If $H$ is finitely generated then it has finite index in $G$.

TheOREM 2. Let $G=A *{ }_{U} B$ (free product of $A$ and $B$ amalgamating the common subgroup $U$ ) and let $H$ be a subgroup of $G$ such that every conjugate $x H x^{-1}$ meets $A$ and $B$ trivially and contains a cyclically reduced word. If $H$ is finitely generated then $G$ has only finitely many $(H, U)$-double cosets.

In Theorem : we fix a free basis $S$ of $G$ and identify elements of $G$ with reduced " $/$ rds in the alphabet $S \cup S^{-1}$. A reduced word $w=s_{1} s_{2} \cdots s_{k}$

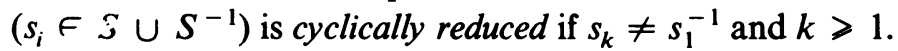

In Theorem 2 by a cyclically reduced word we mean an element $w \in G$ of the form $w=a_{1} b_{1} \cdots a_{n} b_{n}$ or $w=b_{1} a_{1} \cdots b_{n} a_{n}$ where $a_{i} \in A-U, b_{i} \in B$ - $U$ and $n \geqslant 1$.

Theorem 1 should be viewed as a generalization of the well-known result of A. Karrass and D. Solitar [2]: If $H$ is a finitely generated subgroup of a free group $G$ and $H$ contains a nontrivial normal subgroup of $G$ then $(G$ : $H)<\infty$. Indeed, if $N$ is such a normal subgroup then $x H x^{-1} \supset N$ for all $x$ and $N$ contains a cyclically reduced word.

The following result follows immediately from Theorem 2 .

Received by the editors July 13, 1978.

AMS (MOS) subject classifications (1970). Primary 20E05, 20E30; Secondary 05C10, $05 C 25$.

$K e y$ words and phrases. Free group, free product with amalgamation, cyclically reduced words, tree, cycle, fundamental group of a graph.

'This work was supported in part by NRC Grant A-5285. 
COROLlaRY 1. Let $N$ be a nontrivial normal subgroup of $G=A *{ }_{U} B$ such that $N \cap A=N \cap B=\{1\}$. If $N \leqslant H \leqslant G$ and $H$ is finitely generated then $G$ has only finitely many $(H, U)$-double cosets.

It suffices to remark that every element of $G$ is conjugate to one of the following: an element of $A$, an element of $B$, or a cyclically reduced word.

2. Proof of Theorem 1. Let $\Gamma=\Gamma(G, S)$ be the Cayley graph of $G$ with respect to a free basis $S$ of $G$. We refer to [1] or [3] for more details about this graph. $\Gamma$ is a directed graph with vertex-set $G$ and the edge-set $G \times(S \cup$ $\left.S^{-1}\right)$. The origin of an edge $(x, s)$ is $x$ and its terminus is $x s$, where $s \in S \cup S^{-1}$. The opposite of an edge $(x, s)$ is the edge $\left(x s, s^{-1}\right)$ and the orientation of $\Gamma$ is prescribed by the set $G \times S$ as being the positive edges. $\Gamma$ is an infinite tree and $G$ acts on $\Gamma$ freely via left multiplication.

Let $H$ be a subgroup of $G$ and let $X=H \backslash \Gamma$ be the corresponding quotient graph. The vertices of $X$ are the cosets $H a$ and the edges are the sets

$$
\{(h a, s) \mid h \in H\}=(H a, s)
$$

where $a \in G, s \in S \cup S^{-1}$. This edge is positive if $s \in S$.

A cycle in $X$ is a sequence of edges $\left(H a_{1}, s_{1}\right), \ldots,\left(H a_{n}, s_{n}\right)$ such that $H a_{k} s_{k}=H a_{k+1}$ and $\left(H a_{k}, s_{k}\right)$ is not the opposite of $\left(H a_{k+1}, s_{k+1}\right)$ for $1 \leqslant k$ $\leqslant n$ (indices should be reduced modulo $n$ ).

LEMMA 1. The following assertions are equivalent: (i) every vertex of $X$ belongs to a cycle;

(ii) every conjugate of $\boldsymbol{H}$ contains a cyclically reduced word.

Proof. (i) $\Rightarrow$ (ii). Let $x \in G$ and choose a cycle

$$
\left(H x_{1}, s_{1}\right), \ldots,\left(H x_{n}, s_{n}\right)
$$

such that $x_{1}=x$. Since $H x_{1} s_{1}=H x_{2}, H x_{2} s_{2}=H x_{3}, \ldots, H x_{n} s_{n}=H x_{1}$, we have $H x_{1} s_{1} s_{2} \cdots s_{n}=H x_{1}$ and so $w=s_{1} s_{2} \cdots s_{n} \in x_{1}^{-1} H x_{1}$. Since (1) is a cycle, the word $w$ is cyclically reduced and $w \in x^{-1} H x$.

(ii) $\Rightarrow$ (i). Let $x \in G$ and choose a cyclically reduced word $w=s_{1} s_{2} \cdots s_{n}$ in $x^{-1} H x$. Define $x_{1}=x, x_{2}=x_{1} s_{1}, \ldots, x_{n}=x_{n-1} s_{n-1}$. Then (1) is a cycle containing the vertex $H x$.

Now we can prove Theorem 1. Let $T$ be a spanning tree of $X$. Since $H$ acts freely on $\Gamma$, it is isomorphic to the fundamental group $\pi_{1}(X)$ of $X$. Using that $H$ is finitely generated, we deduce that there are only finitely many edges of $X$ outside $T$. Since (by Lemma 1) every vertex of $X$ belongs to a cycle, it follows that $X$ is finite. Thus $(G: H)<\infty$.

3. Proof of Theorem 2. Let $\Gamma$ be the directed graph whose vertex-set is the disjoint union of $G / A$ and $G / B$ and with $G / U$ as the set of positive edges. The origin of an edge $a U$ is the vertex $a A$ and its terminus the vertex $a B$. The opposite of a positive edge $a U$ will be denoted by $\overline{a U}$. 
The group $G$ acts on $\Gamma$ via left multiplications and preserves the orientation of $\Gamma$. Of course this action is not free because for instance, the subgroup $A$ is the fixer of the vertex $1 \cdot A$.

Let $H$ be a subgroup of $G$ such that every conjugate $x H x^{-1}$ has trivial intersection with $A$ and $B$. This condition is equivalent to the condition that the action of $H$ on $\Gamma$ be free. Let $X=H \backslash \Gamma$ be the corresponding quotient graph. We may identify the vertices of $X$ with the disjoint union of the sets of double cosets $H \backslash G / A$ and $H \backslash G / B$ and the set of positive edges with $H \backslash G / U$. The origin of the positive edge $H a U$ is $H a A$ and its terminus is $H a B$. The opposite of the edge $H a U$ we shall denote by $\overline{H a U}$.

A cycle in $X$ is a sequence of edges

$$
H x_{1} U, \overline{H y_{1} U}, \ldots, H x_{n} U, \overline{H y_{n} U}
$$

such that $H x_{k} B=H y_{k} B, H y_{k} A=H x_{k+1} A$, and $H x_{k} U \neq H y_{k} U \neq H x_{k+1} U$ for $1 \leqslant k \leqslant n$ (indices to be reduced modulo $n$ ).

LEMMA 2. The following assertions are equivalent: (i) every edge of $X$ belongs to a cycle;

(ii) every conjugate of $H$ contains a cyclically reduced word.

Proof. (i) $\Rightarrow$ (ii). Let $x \in G$ and choose a cycle

$$
H x_{1} U, \overline{H y_{1} U}, \ldots, H x_{n} U, \overline{H y_{n} U}
$$

such that $x_{1}=x$. Since $H x_{1} B=H y_{1} B$ we may assume that $y_{1}=x_{1} b_{1}$ with $b_{1} \in B$. Since $H x_{1} U \neq H y_{1} U=H x_{1} b_{1} U$ we infer that $b_{1} \in B-U$. From $H y_{1} A=H x_{2} A$ we deduce that we may assume that $x_{2}=y_{1} a_{1}$ where $a_{1} \in A$. Since $H y_{1} U \neq H x_{2} U$, we obtain that $a_{1} \in A-U, x_{2}=x b_{1} a_{1}$. Continuing this argument, we obtain $y_{n}=x b_{1} a_{1} \cdots a_{n-1} b_{n}$ with $a_{i} \in A-U, b_{i} \in B-$ $U$. Since $H y_{n} A=H x_{1} A$ we obtain $x=h y_{n} a_{n}$ where $h \in H, a_{n} \in A$. From $H y_{n} U \neq H x_{1} U$ we obtain that $a_{n} \notin U$. Hence we have $w=x^{-1} h^{-1} x=$ $b_{1} a_{1} \cdots b_{n} a_{n} \in x^{-1} H x$ and $w$ is cyclically reduced.

(ii) $\Rightarrow$ (i). Let $x \in G$ and choose a cyclically reduced word $w=$ $b_{1} a_{1} \cdots b_{n} a_{n}$ in $x^{-1} H x$ (note that if a cyclically reduced word begins with an $a$ then its inverse is also cyclically reduced and begins with a $b$ ).

Define $x_{1}=x, y_{k}=x_{k} b_{k}(1 \leqslant k \leqslant n), x_{k+1}=y_{k} a_{k}(1 \leqslant k \leqslant n-1)$. Then (2) is a cycle in $X$ containing the edge $H x U$. By replacing each edge in (2) by its opposite and reversing their order we obtain a cycle containing the edge $\overline{H x U}$.

Now we can prove Theorem 2 . Since $H$ acts freely on $\Gamma$ we can identify $H$ with the fundamental group $\pi_{1}(X)$ of $X$. Let $T$ be a spanning tree of $X$. Since $H$ is finitely generated, there exist only finitely many edges of $X$ outside $T$. Since by Lemma 2 every edge of $X$ belongs to a cycle, we deduce that $X$ must be finite, i.e., there are only finitely many $(H, U)$-double cosets in $G$. 


\section{REFERENCES}

1. W. Imrich, Subgroup theorems and graphs, Proc. Fifth Australian Conf. on Combinatorial Math., Lecture Notes in Math., vol. 622, Springer-Verlag, Berlin and New York, 1977, pp. 1-27.

2. A. Karass and D. Solitar, Note on a theorem of Schreier, Proc. Amer. Math. Soc. 8 (1957), 696-697.

3. J.-P. Serre, Arbres, amalgames, $\mathrm{SL}_{2}$, Astérisque, No. 46 (1977).

Department of Pure Mathematics, Universtry of Waterloo, Waterloo, Ontario, Canada N2L 3G1 